\title{
INCREASING ROBUSTNESS OF TIMETABLES BY DELIBERATE OPERATION OF TRAINS TO SHORTEN HEADWAYS
}

\author{
H. YABUKI ${ }^{1,2}$, Y. TAKATORI ${ }^{1}$, M. KORESAWA ${ }^{1}, \&$ N. TOMII ${ }^{2}$ \\ ${ }^{1}$ Tokyo Metro Co. Itd., Japan. \\ ${ }^{2}$ Department of Computer Science, Chiba Institute of Technology, Japan.
}

\begin{abstract}
In railway lines where trains are running densely, a small increase of a dwell time causes a delay and the delay tends to expand and propagate to many other trains. One idea to avoid such delays is to increase dwell times of trains in timetables. Increase of dwell times, however, may cause an expansion of headways and we may have to decrease the frequency, which is never allowed in a congested railway line. In this article, we propose a procedure to get a timetable in which each train is given an enough amount of dwell time without reducing the frequency of trains. Our key idea is to find a train performance curve which minimizes the departure-arrival interval of trains and to make use of the generated time to increase the dwell times. In this process, we do not stick to an idea that travel times of trains have to be minimized. Following this procedure, we can increase the dwell times without reducing the frequency. We have applied our procedure to actual timetables and confirmed that our procedure works very well. Keywords: headway, robustness, timetable, train performance curve.
\end{abstract}

\section{INTRODUCTION}

In Tokyo, the capital of Japan, a great number of people commute using railways. In order to fulfil the big demand for railways, trains are operated very densely. As a matter of fact, during morning rush hours, 25 to 30 trains are running per hour per direction on a double track in many railway lines in Tokyo. Trains typically consist of 8 to 10 cars, which are $160 \mathrm{~m}$ to 200 $\mathrm{m}$ long. Nevertheless, congestion rates of trains are very high. It is not unusual to find trains during rush hours of which the congestion rates are more than $150 \%$, which means more than 2,000 passengers are on board.

One of the recent problems in the railway network of Tokyo area is that short delays very often happen during morning rush hours. Small, say several minutes, delays happen almost every weekday in the morning.

The main cause of such delays is an excess of dwell times. If more passengers than expected get on and/or off, dwell times increase. It is often observed that a passenger's bag or an umbrella is caught by doors of a train due to congestion inside the train. In such cases, the doors must be opened again and the dwell time increases. Another case is rescue of a sick passenger. If a passenger gets sick inside a train, he/she is rescued by an ambulance and the dwell time increases.

One of the characteristics of railway systems in Tokyo area is that so-called direct operation is widely realized. This is an operation which allows trains from suburban areas go directly into subway lines and vice versa. Passengers can arrive at downtown without transferring,

This paper is part of the proceedings of the 15th International Conference on Railway

Engineering Design and Operation (COMPRAIL)

www.witconferences.com 
which is very convenient for them. On the other hand, the direct operation has a drawback that a delay tends to propagate to other railway lines. Thus, railway companies are keen to prevent such delays from happening and making every effort to make their timetable more robust [1-3].

In order to make timetables more robust, it is effective to increase dwell times in the timetables. But the problem is that because we are required to set up a certain number of trains to fulfil the demands, it is impossible to increase the dwell times any more, which we will discuss later.

In this article, we introduce a procedure to increase dwell times of trains for a given timetable without decreasing the frequency of trains (we define 'a frequency' as the number of trains during rush hours in this article). If we can increase the dwell times, delays due to an increase of dwell times do not occur anymore and the timetable becomes more robust. Our key idea is to find a train performance curve which minimizes the arrival-departure interval of trains and to make use of the generated time to increase the dwell times. In this process, we do not stick to an idea that travel times of trains have to be minimized.

The process to seek for such a train performance curve is not so simple. One reason is that we have to find a practical performance curve. We have to be careful about the ride comfort and should avoid losses. It is not desirable if it is difficult to drive. Another reason is that we have to assure that the performance curve has to be applicable for all the trains, because it is not reasonable to specify different performance curves for each train. One more reason is that we have to consider an influence from the preceding train because trains are running densely. These mean that in the procedure, we have to consider the preceding train, the current train and the succeeding train all at the same time. To solve the problems, we introduce a procedure which consists of iteration and feedback.

We have applied our procedure to an actual timetable and confirmed that our procedure successfully increases the dwell times of a given timetable.

\section{MAKING A TIMETABLE}

\subsection{Constraints in making a timetable}

To make a timetable means fixing the arrival and departure times of trains at stations together with finding out whether trains pass or stop at stations and the track which the trains are going to use. In Tokyo Metro, arrival and departure times are specified with a unit of 5 seconds.

There exist three major constraints we have to take into account when we make a timetable.

1. Minimum running time

A minimum running time of a train means the minimum time required when a train runs from a station to the next station. It is common that we consider only one train and we do not care about an interaction between two trains. This is because what we want to get here is the minimum running time when a train runs without any interference from other trains.

Minimum running times are nowadays obtained by calculation using a computer software. The output of the software is a so-called train performance curve (hereafter, we may call it just 'a performance curve' if there is no ambiguity), which consists of the speed curve and the time curve. We show an example of a train performance curve from one station (Station A) to the next station (Station B) in Fig. 1. The $\mathrm{x}$-axis is the distance and the $\mathrm{y}$-axis is the speed and the time. The blue line expresses the speed limit imposed by ATP. The speed 


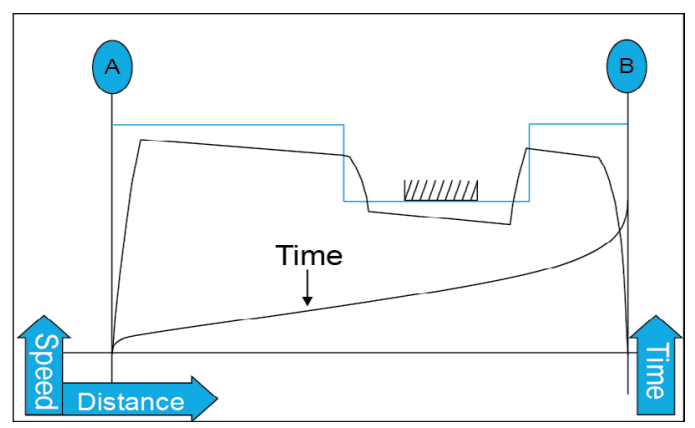

Figure 1: Train performance curve (example).

curve depicts the running speed of the train at each location together with how the train should be driven, namely, where the train should accelerate and where the train should decelerate. From the time curve, we can know how much time is needed for the train to run between the stations. The unit of time for the results of calculation is usually 1 second and is rounded to a time with a unit of 5 seconds in Tokyo Metro (and in other railway companies in Japan). Hereafter, we call the rounded time a technically minimum running time and if there is no ambiguity we just call it a minimum running time.

2. Dwell times

We have to set the dwell time of trains at each station. We estimate or observe how many passengers get on/off at the station and fix an appropriate dwell time. Thus, the dwell times vary according to the time, the direction and the station. Dwell times during rush hours are set to be longer than those in less congested time. In Tokyo Metro, dwell times are typically from 30 seconds to 1 minute.

3. Minimum headway

When two trains consecutively use the same track at a station, we have to keep a certain time interval between these two trains (it is true that we also have to consider the time interval outside a station. But because the necessary interval outside stations is usually larger than that of inside a station, we focus on the necessary interval inside a station). In this article, we call the minimum time interval between two trains which consecutively run 'a technically minimum headway' or 'a minimum headway' if there is no ambiguity. More exactly speaking, the minimum headway in this article is defined as the minimum necessary time from the departure of a train to the arrival of the next train (Sometimes, a headway is thought to be an interval of trains in the timetable. But in this article, we use 'headway' as the minimum interval as described above. We do not want to change the frequency of trains. This is the most important prerequisite of this article.).

The minimum headway is influenced by the signalling system (ATP) to a large extent. There are no wayside signals in Tokyo Metro and the allowable speed at that moment is shown in the cab of the train. The signal continuously changes as the situation such as the location of the preceding train changes.

Minimum headways are also calculated by computers nowadays. The computer software calculates the minimum headways using the train performance curves and outputs a picture called a minimum headway chart as shown in Fig. 2. Unlike the train 


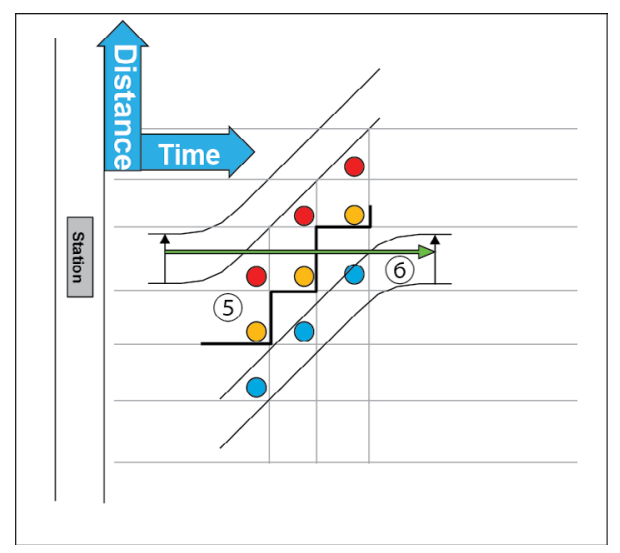

Figure 2: A minimum headway chart (example).

performance curve, the $\mathrm{x}$-axis is the time and the $\mathrm{y}$-axis is the distance. The minimum headways are also rounded with a time unit of 5 seconds in Tokyo Metro.

We would like to explain how Tokyo Metro is dealing with the margins in running times when we make a timetable. We understand it is common to include some amount of margin in the running times of trains in timetables. Namely, in a timetable, the following formula holds:

Arrival time of a train at a station - departure time of a train at the prior station $=$ technically minimum running time + margin

In Tokyo Metro (and many other railway companies in Japan which operate trains in urban area), however, we do not give a margin explicitly. Instead, we impose rather strict conditions when we calculate the minimum running times (such as the voltage of the catenary, the diameter of the wheels and so on) and we expect some margin is included in the minimum running time eventually. This is because in Tokyo Metro, distances between stations are rather short and the types of the trains are almost the same and it is not necessary to give different amount of margins to trains. Thus, it is more reasonable that a certain amount of margin is implicitly included in the minimum running times.

\subsection{Conventional Process to make a timetable}

When a railway line is newly constructed, we have to make a timetable from scratch. The basic process in this case is as follows:

1. From the forecast of demands, we fix the necessary frequency of trains during the busiest hours.

2. We draw a train performance curve to get the minimum running times.

3. We fix necessary dwell times for each station based on an estimation of the number of passengers. Dwell times may be different depending on times of the day, direction, etc.

4. We design the facilities including signalling systems so that we can realize the minimum running times and the frequency during the busiest hours.

5. We fix arrival and departure times of each train. 


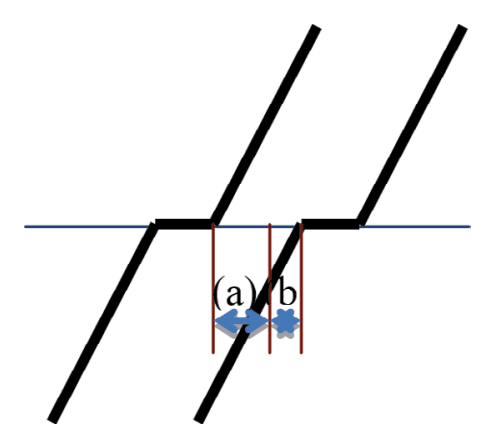

Figure 3: Dwell time, headway and buffer time.

When we want to revise an existing timetable, minimum headways and minimum running times become constraints. If necessary, we might change the facilities so that we can relax these constraints. But this is usually very difficult or almost impossible because the facilities such as signalling systems are already optimized and further improvement is not possible any more.

We have to note that this process is aiming at reducing the travel time and so much attention was not paid to increasing the dwell times.

\subsection{Current issues concerning timetables}

As we have discussed before, the major cause of short delays is an increase of dwell times. In order to prevent such delays, dwell times should be long enough in the timetable. But we cannot make the dwell time longer unless we decrease the frequency. This is because we have to consider the constraint of the minimum headway and if we increase dwell times, it becomes impossible to keep the frequency of trains. Figure 3 expresses the reason. In Fig. 3, (a) means the minimum headway and (b) is a buffer time. The dwell time could be increased by (b) (the buffer time), but if we make the dwell time longer than this, we have to change the arrival time of the next train due to the constraint of the minimum headway. This means the frequency decreases. Trains are very congested; so it is never allowed to reduce the frequency. In addition, in the timetables of Tokyo Metro, dwell times are already prolonged almost to the limit and it is almost impossible to increase the dwell times any more. Hence, we need to introduce a brand new idea to increase the dwell times in the timetable.

\section{INCREASING ROBUSTNESS OF TIMETABLES BY DELIBERATE OPERATION OF TRAINS TO SHORTEN HEADWAYS}

\subsection{Basic ideas}

As discussed above, it is difficult or almost impossible to increase dwell times in the timetables. But it might be possible to increase dwell times if we can reduce minimum headways. If a minimum headway becomes smaller, we can increase the dwell time without reducing the frequency by making use of the generated time. Figure 4 explains the idea. If we can reduce the headway to $\left(a^{\prime}\right)$, we can increase the dwell time by $(c)$ at the largest, which is calculated as

$$
(c)=(b)+\left((a)-\left(a^{\prime}\right)\right) \text {. }
$$




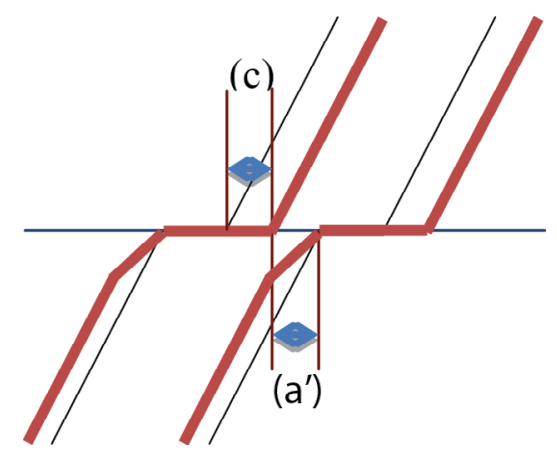

Figure 4: To increase dwell time by reducing headway.

In order to reduce minimum headways, we have to allow trains enter a station with a lower speed. Conventionally, we assume a train runs seeing a green signal (this is a kind of metaphor. As we have explained, in Tokyo Metro, there are no wayside signals) when we make a train performance curve. This is because we want to get the 'minimum' running time. We change this assumption so that we admit a train sees an aspect other than green. Thus, trains may run with a lower speed and the running time may increase, which might be regarded that the level of service is degraded. But we believe punctuality is more important and it is all right if we can increase robustness by slightly making the travel times longer.

\subsection{Issues}

In order to implement this idea, it is indispensable to establish a procedure to find a train performance curve which realizes the minimum interval. In establishing this procedure, there exist three issues to be settled.

1. The train performance curve must be feasible from practical point of view.

This means that we have to consider the ride comfort for one thing. In addition, it is not preferable if it is difficult to drive. For example, a sudden change of driving mode is not desirable. We also should consider avoiding losses. For example, if there exists a speed limit ahead, the train should not accelerate and hit the brakes but should coast.

2. The same train performance curve has to be applicable to all the trains for the segment (in this article, we define a segment as a track between a station to the next station).

Trains are driven by drivers. We have to indicate them how to drive a train in advance. It is not reasonable to prepare different train performance curves for each train but we have to prepare only one train performance curve which all trains can follow.

3. We have to consider the preceding train at the same time.

In a railway line where trains are operated densely, when a train is arriving at a station, the signal aspect for the train is influenced by the preceding train which is just departing from the station. Namely, we have to make the train performance curve considering the current train and the preceding train at the same time.

Although there exist a lot of papers concerning algorithms to make train performance curves, none of them deal with these three issues [4]. 


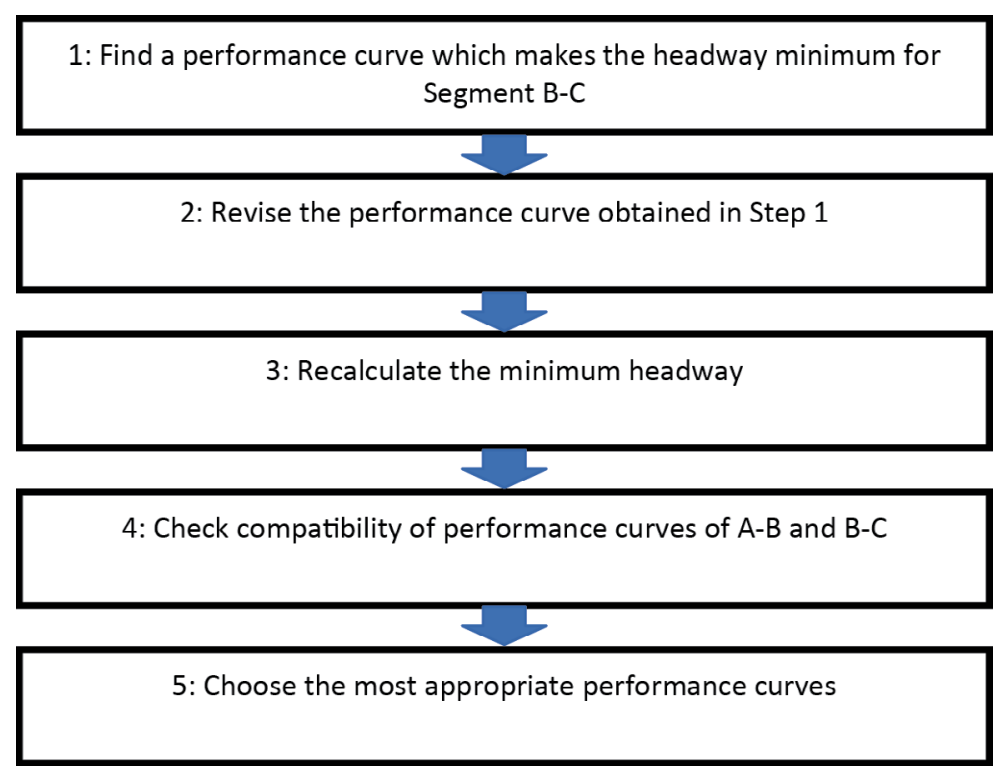

Figure 5: Overall structure of the procedure.

\subsection{Procedure}

We show our procedure to get a timetable in which dwell times are made longer. The overall structure of the procedure is depicted in Fig. 5. The procedure is basically an iteration of making and confirming the train performance curves, in which a feedback to check the mutual influence is included. More exactly speaking, we try to find a performance curve for one segment and we iterate this process to the following segments. There exist a couple of problems inherent in this process. The first one is that the performance curve obtained in a segment is not applicable to the succeeding train in the same segment. The succeeding train cannot always run as specified by the performance curve obtained. The second problem is that it may reveal that a performance curve obtained for the next segment is not compatible with the performance curve obtained for the current segment. So, during the procedure, we occasionally confirm these compatibilities and if some incompatibility is found, we modify the performance curves so that we can assure the compatibility.

For simplicity, we show a procedure in which only three stations appear: Station A, Station B and Station C (trains proceed from Station A to Station C) and we want to increase the dwell times at Station C. By iterating this procedure, however, we can deal with more complicated situation such as more stations are involved and dwell times of more than one station are increased.

Step 1: Find a performance curve which makes the headway minimum for Segment B-C We first try to find a train performance curve from Station B to Station C (Segment B-C) which realizes the minimum headway when trains arrive at Station C. Such performance curve must exist between a performance curve for the minimum running time and a performance curve in which the train is almost compelled to stop before it arrives at Station C. We enumerate all train performance curves by fixing the preceding train and changing the departure time of a train at Station B by 1 second and choose one in which 
the interval between the departure of the preceding train at Station $\mathrm{C}$ and the arrival of the train at Station $\mathrm{C}$ is the smallest.

\section{Step2: Revise the performance curve obtained in Step 1}

The train performance curve generated in Step 1 is not always appropriate from practical point of view. So we modify it manually so that the performance curve is practically appropriate as discussed earlier.

We also confirm if the obtained performance curve can be applied by the succeeding train(s) as well. We simulate the movement of one train (the first train) and the succeeding train simultaneously and confirm if the succeeding train can run following the same performance curve. If not, we modify the performance curve manually so that the trains can run following the same performance curve.

\section{Step 3: Recalculate the minimum headway}

If we have modified the performance curve in Step 2, this means the modified performance curve does not always realize the minimum headway. Hence, we calculate the interval between the train (the first train) and the succeeding train based on the modified performance curve. In this process, we confirm how closely the succeeding train can run considering the signals which are influenced by the first train.

\section{Step 4: Check compatibility of performance curves of A-B and B-C}

Now, we may have a new performance curve for Segment B-C. This means that trains may not be able to run in Segment A-B according to the current performance curve. This is because when a train is arriving at Station B, the preceding train is departing from Station B based on the modified performance curve and the signal aspects for the train are influenced by the preceding train. Thus, we have to check the compatibility of performance curve for Segment A-B and that for Segment B-C. In order to check the compatibility of the performance curves, we simulate two trains for these two segments simultaneously.

\section{Step 5: Choose the most appropriate performance curves}

Here, we may have the following four options.

If the two performance curves are proved to be compatible in Step 4,

(1) we can just adopt the current performance curve for Segment A-B and the obtained performance curve for Segment B-C.

If the performance curves are proved to be incompatible,

(2) we modify the performance curve for Segment A-B (we do not change the current performance curve for Segment B-C), which we can obtain by applying a procedure similar to Steps 1-3 to Segment A-B.

(3) we modify the performance curve for Segment B-C (we do not change the current performance curve for Segment A-B). We can accomplish this by paying attention to the change of the signal aspects and by modifying the performance curve for Segment B-C so that it does not give an influence to the succeeding train in Segment A-B.

(4) we change the performance curves for Segment A-B and Segment B-C. This could be done as a combination of the two procedures described above.

Then, we choose one of these options which we believe is the most appropriate for the situation. 


\section{NUMERICAL EXPERIMENTS}

We have conducted numerical experiments using actual data. The data we used are ones of Hibiya line of Tokyo Metro. We intended to get a timetable in which dwell times at Ueno station (this is the busiest station in Hibiya line. Hereafter, we call the station 'Station U') are increased. The station prior to Ueno is Iriya (hereafter, 'Station I') and the station prior to Iriya is Minowa (hereafter, 'Station M'). Station U corresponds to Station C, Station I corresponds to Station B and Station M corresponds to Station A, respectively, in the procedure we showed above.

We show the results in Table 1. It was proved that we cannot realize option (1). Namely, if we change the performance curve for Segment B-C, trains cannot run according to the current performance curve for Segment A-B. Hence, we have three options (2), (3) and (4) as shown in Table 1.

Now, we examine and compare these options from various kinds of viewpoints such as whether the riding comfort is considerably good, whether it is not difficult to drive, how much the travel time increases, how much we can increase the dwell times and so on. If we examine the above three options, the discussions are as follows:

- If we adopt option (2), we can increase the dwell time of Station U by 10 seconds. But we are concerned that drivers have to rather suddenly change the speed in Segment M-I because the signalling system indicates a low speed limit for a short time.

- For option (3), it is preferable that the performance curve for Segment M-I is the same as the current one but the increase of the dwell time at Station $U$ is the smallest. The increase of the travel time is the smallest.

- We can get the largest increase of the dwell time at Station U if we adopt option (4).

So one conclusion might be that if we do not care to introduce new performance curves, we should adopt option (4). But if we prefer not to change the performance curve for Segment $\mathrm{M}-\mathrm{I}$ and if we do not like the running time increases so much, we should adopt option (3).

Table 1: Results of the numerical experiments

\begin{tabular}{|c|c|c|c|c|c|c|c|}
\hline & \multicolumn{2}{|c|}{$\begin{array}{l}\text { Heaway } \\
\text { (sec) } \\
\end{array}$} & $\begin{array}{l}\text { Invease } \\
\text { Invel (sec) }\end{array}$ & \multicolumn{4}{|c|}{ Pertoumance cuve, Rurning time } \\
\hline & u- & HU & v & M-I & & I-U & \\
\hline anment & 71 & 60 & & 90 sec. & & $95 \mathrm{sec}$. & \\
\hline$\Rightarrow$ & - & - & & & & possitle & \\
\hline (2) & 71 & 50 & +10 & 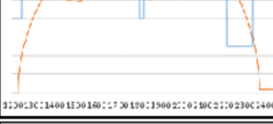 & \begin{tabular}{|l}
89 ser \\
ni-1 setl
\end{tabular} & & \begin{tabular}{|l}
$125 \mathrm{sex}$ \\
$\mathrm{a}+30 \mathrm{sec} \|$
\end{tabular} \\
\hline (3) & 71 & 52 & +8 & 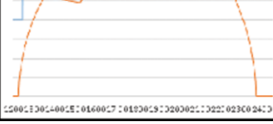 & \begin{tabular}{|c|}
90 ser \\
let secu
\end{tabular} & 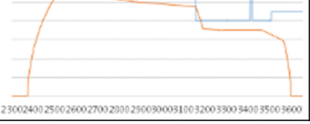 & \begin{tabular}{|l}
$114 \mathrm{sec}$ \\
$\mathbf{u}+19 \sec \|$
\end{tabular} \\
\hline (4) & 71 & 48 & +12 & II & $\left|\begin{array}{c}28 \sec \\
0-2 \sec \|\end{array}\right|$ & $\sum_{201}$ & $\begin{array}{l}119 \sec \\
0+24 \sec \end{array}$ \\
\hline
\end{tabular}


It is a great merit of our procedure that it can show several options and leaves a freedom of choice for the users to examine them from various kinds of viewpoints.

\section{CONCLUSIONS}

We have proposed a procedure to increase dwell times for a given timetable without reducing frequency. The key idea of the procedure is to find a train performance curve which realizes the minimum headway. In this process, we do not stick to an idea to make trains run as fast as possible but we rather decrease the running speed so that we can obtain a performance curve which makes the headway minimum. Thus, trains may enter with a lower speed but the headway becomes smaller. If the headway becomes smaller, we can consume the generated time to increase the dwell times.

Based on this idea, we have introduced a procedure with which we can know how we can increase the dwell times in a timetable. The procedure could show several alternatives. The outputs contain a performance curve and other figures such as how much we can increase the dwell times, how much the running times increase and so on, which are useful information for users. We have examined how the procedure works using actual data and confirmed that our procedure works very well.

Some of our future works are the following:

- Application of this idea to real-time environment.

We have introduced a procedure to revise a timetable so that the train can have longer dwell times. It is useful to give an indication to a driver to run so that the train can arrive with a minimum headway when the dwell time of the preceding train has become longer and is delayed.

- Apply this idea when renovation of the signalling systems is allowed.

In this article, we assumed that we do not change the signalling systems. But it is more effective if we can renovate the signalling system because signalling systems are very influential to headways.

\section{ACKNOWLEDGEMENTS}

This research is partly supported by the Ministry of Education, Science, Sports and Culture, Grant-in-Aid for Scientific Research (C) 15K01199.

\section{REFERENCES}

[1] Yamamura, A., Koresawa, M., Adachi, S. \& Tomii, N., How we have succeeded in regaining Punctuality in Tokyo Metropolitan Railway Network? WCRR2013-World Congress on Railway Research, Sydney, Australia, 2013.

[2] Yamamura, A., Koresawa, M., Adachi, S. \& Tomii, N., Taking effective delay reduction measures using delay elements of indices on the Tokyo metropolitan railways. COMPRAIL 2014, Rome, Italy, 2014.

[3] Ochiai, Y. \& Tomii, N., Punctuality analysis using a microscopic simulation in which drivers' behaviour is considered. Journal of Rail Transport, Planning and Management, 5(3), pp. 128-145, 2015.

[4] Goodman, C., Overview of electric railway systems and the calculation of train performance. 2008 IET Professional Development course on Traction Systems, Manchester, Institution of Engineering and Technology, 2006. 